\title{
Margaret Wertheim: Complexity, Evolution and Hyperbolic Space
}

\author{
Mick Wycoff
}

Published online: 25 September 2008

(C) Springer Science + Business Media, LLC 2008

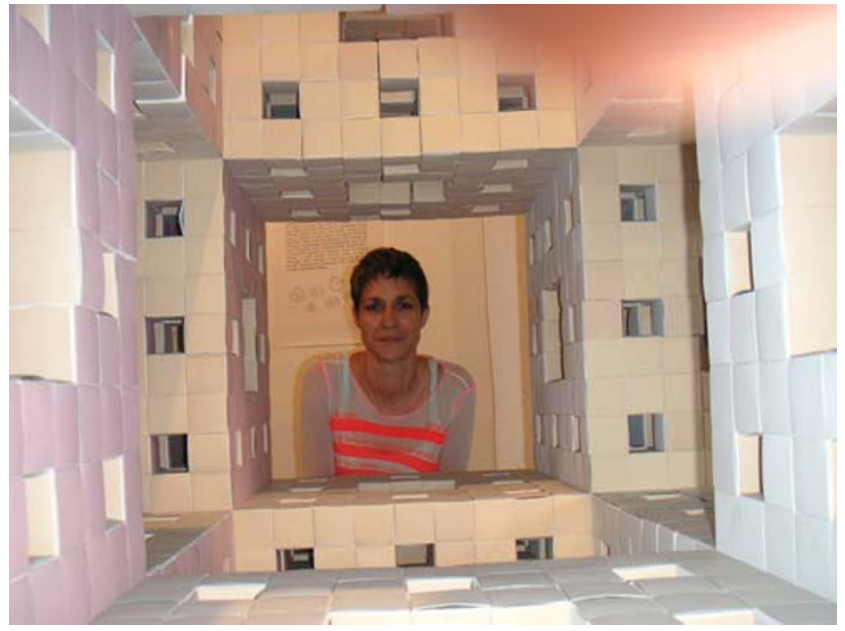

Margaret Wertheim looking through the Business Card Menger Sponge. The BCMS is a the giant origami fractal made by engineer Dr Jeannine Mosely and exhibited by the Institute For Figuring. More information at the IFF online exhibit: http://www.theiff.org/oexhibits/ menger01.html

Margaret Wertheim earned degrees in physics and mathematics in her homeland, Australia, before becoming a prize-winning science journalist and author. She is the founder, with her twin sister, Christine, of the Institute For Figuring, a new kind of "feral science" organization located in Los Angeles. The IFF's mission is to promote the public understanding of the poetic and aesthetic dimensions of science and mathematics. Wertheim's books include Pythagoras' Trousers, a history of the relationship between physics and religion

M. Wycoff $(\bowtie)$

The Eldredge Group,

Ridgewood, New Jersey, USA

e-mail: meldredge@earthlink.net
(1995), The Pearly Gates of Cyberspace: A History of Space from Dante to the Internet (1999) and A Field Guide to Hyperbolic Space (2005), upon which this interview was based.

Why are you fascinated with hyperbolic space?

Because it gives us important new insights into nature and the cosmos.

We have built a world of rectilinearity: the rooms we inhabit, the skyscrapers we work in, the grid-like arrangement of our streets and the freeways we travel on our way to work speak to us in straight lines.

People have learned to play by Euclidean rules because 2,000 years of geometrical training have engraved the grid in our minds. But in the early nineteenth century, mathematicians became aware of another kind of space in which lines cavorted in strange and seemingly aberrant formations. This suggested the existence of a new geometry. To everyone at the time, hyperbolic space seemed pathological, because it didn't conform to one of Euclid's most cherished principles, the so-called parallel postulate. In this sense, it contradicted millenia of mathematical wisdom and, frankly, it offended common sense.

But eons before the dawning of mathematical awareness, nature had exploited this supposedly forbidden form, realizing it throughout the vegetable and marine kingdoms. Outside our boxes, the natural world teems with swooping, curling, crenellated forms, from the fluted surfaces of lettuces and fungi to the frilled skirts of nudibranchs and sea slugs and anemones. Nature just loves hyperbolic structures.

What is also fascinating is that although physicists had long thought the space of our universe must necessarily conform to Euclid's ideals, data coming from telescopic studies of the early universe suggest the cosmological whole may possibly be a hyperbolic form.

How does the concept of hyperbolic space shed light on complexity? 
Let us start with Euclid's fifth axiom regarding parallel lines (this is also known as the parallel postulate). The postulate states that, given a straight line and an external point, there is no more than one line you can draw through the point which will never meet the original line. All other lines will slant with respect to this line and eventually intersect it. Although this seems intuitively correct, it's actually far more complicated than Euclid's other axioms and, from the start, mathematicians felt haunted by the need for a less complex articulation. If the proposition is really true, they felt, then ought it not be provable from other, simpler axioms (Fig. 1)?

If you think there isn't a problem here, then consider the example of a sphere. That's a surface which forms a geometry very different to the plane because now you're talking about a curved surface. Immediately, we are faced with a question: what does it mean to talk about straight lines in a curved space? Mathematically, a straight line may be generalized to the concept of a geodesic, a term that defines the shortest path between two points. Airlines use such geodesics when charting the paths of international flights, which often look curved on a flat map but are "straight" in relation to the globe itself (Fig. 2).

With respect to a sphere, we notice that the geodesics are not infinite, as they are on a plane, but finite-inevitably connecting back up on themselves. Thus, on the surface of a sphere, there are no straight lines through a point that do not meet the original line. Whereas, on the plane, there is always one non-intersecting straight line; now, we have a geometry in which all lines meet. Still, Euclid's postulate holds since it states there can never be more than one line through a point that doesn't meet an original line, and on a sphere there are none.

But how do we know there isn't some other surface in which there may be two or even more parallels?

The idea that one might not be the limit struck terror into mathematicians' hearts, and evoked an almost moral outrage. For hundreds of years, they sought to prove that any alternative to the single parallel line model was impossible - they really thought that if the parallel postulate was violated and more than one parallel was allowed, logical chaos would ensue. Finally, in the nineteenth century, this effort exhausted itself and mathematicians accepted that the
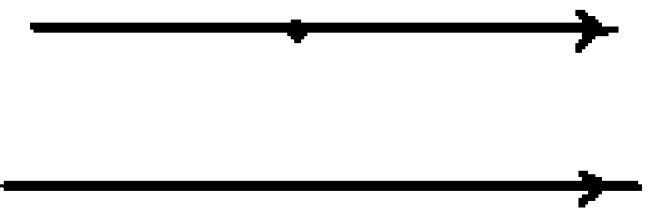

Fig. 1 A Euclidian diagram of parallel lines. Credit: Institute For Figuring

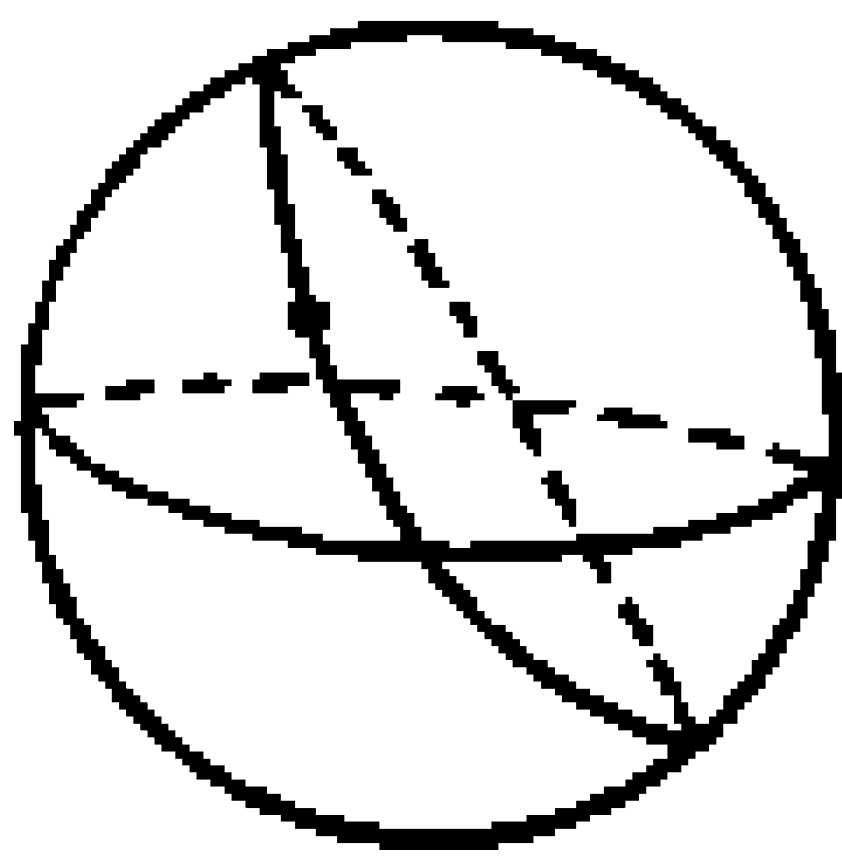

Fig. 2 A diagram of geodesic lines on a curved surface. Credit: Institute For Figuring

parallel postulate actually isn't necessarily true. It is true on an ordinary plane, but it isn't true "always."

So what exactly is hyperbolic space?

To put it into the terms of the nineteenth-century English mathematician John Playfair, there exists a space in which, given a line and an external point, there is actually a multitude of other straight lines that intersect with the point, yet do not meet the original line. Instead of there being just one parallel, there are many. In fact, there are infinitely many. Bizarre though this may seem, the situation gives rise to a consistent geometry. In homage to this abundance of parallels, mathematicians named it the hyperbolic plane.

At this point, readers may object that the lines in the illustration depicting hyperbolic space (below) don't look straight — but that's just because we are trying to see them from our limited Euclidean perspective. From the point of view of someone inside the hyperbolic surface, all these lines would be perfectly straight and none would meet the original line (Fig. 3).

Still, it is one thing to know that something is logically possible and quite another to understand it. Like the blind man and the elephant, hyperbolic space appears in different guises depending on how we approach it. Late in the nineteenth century, the French mathematician Henri Poincaré devised a model of the entire hyperbolic plane within a circular disc. In reality, the hyperbolic plane is infinitely largelike the Euclidean plane, it goes on forever. So we must make some compromises to view it within our Euclidean framework. The Poincaré compromise is to represent angles truly_-but while distorting scale. 


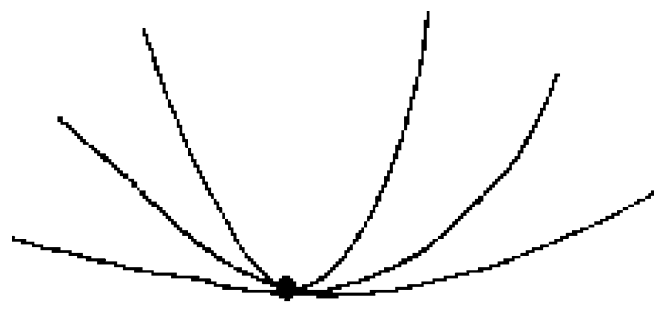

Fig. 3 A diagram of parallel lines in hyperbolic space

Regarding the Poincaré disc model from our Euclidian perspective, the triangles appear to be decreasing in size as we move towards the perimeter. But viewed from inside the disc, every triangle is the same size. Identical, equal-sided triangles stretch endlessly outwards towards an infinite boundary (Fig. 4).

The twentieth century Dutch artist, M.C. Escher, who learned of hyperbolic space from the Canadian geometer Donald Coxeter, explored the endless symmetries inherent in the hyperbolic plane with a series of wonderful illustrations. In Circle Limit III, red, green, blue, and yellow fish splash about in a symphony of triangles and squares. In Circle Limit IV, angels and demons flutter in a hyperbolic trinity, fanning out from a central point to fill the space with hexagons and octagons. Again, within their Poincaré discs, the angels and demons and fish fill their worlds to the bounds of infinity if only we step into the space with them (Figs. 5 and 6)

Isn't there a more accurate, concrete way to visualize hyperbolic space?

Up to nearly the end of the twentieth century, most mathematicians believed it was impossible to construct physical models of hyperbolic forms - in spite of the fact that nature had been doing just that for hundreds of millions of years in the production of such life forms as kelps, corals, sponges, and nudibranchs.

Then in 1997, Latvian mathematician Dr. Daina Taimina finally worked out how to make a model of hyperbolic space with crochet. She based her discovery on a suggestion that had been put forward in the 1970s by the great American geometer William Thurston. He noted that one of the qualities of hyperbolic space is that as you move away from any point, the space around it expands exponentially. He was able to model a small part of this using paper anulii; however, these models were hard to make, hard to handle, and inherently fragile.

Having spent her childhood steeped in feminine handicrafts, Taimina realized that the essence of this construction could be implemented with crochet by increasing the number of stitches in each row. As one increases, the surface naturally begins to ruffle and crenellate. The beauty of her method is that many of the intrinsic properties of hyperbolic space become visible and can be directly experienced by playing with these models. They're really very tactile forms.

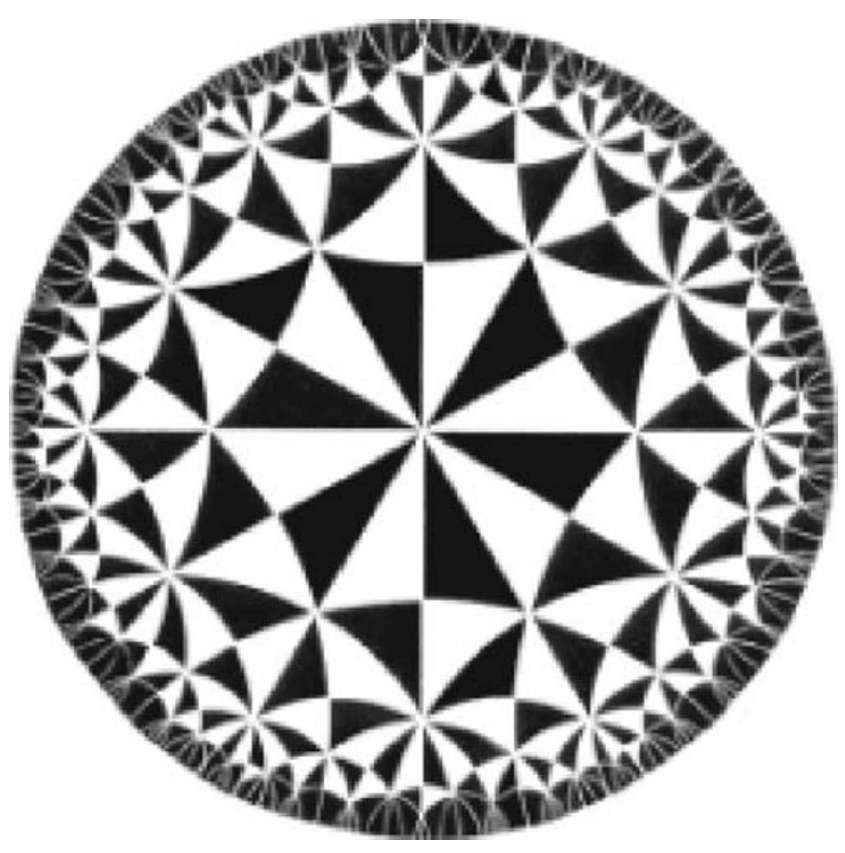

Fig. 4 A diagram of the Poincare disc model. Credit: Institute For Figuring

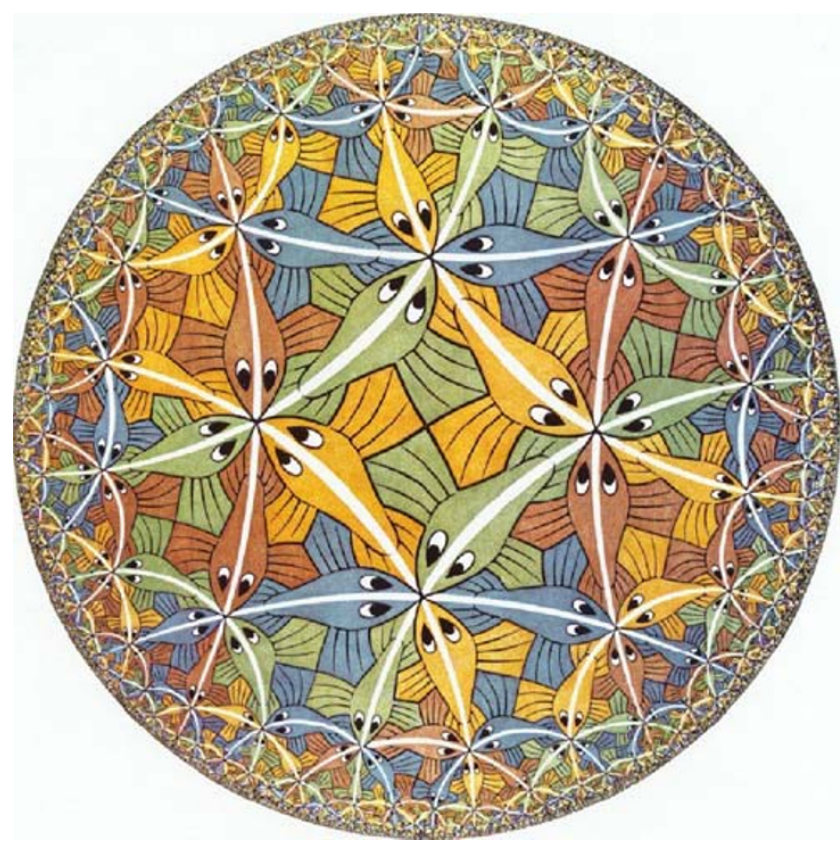

Fig. 5 Circle Limit III, M.C. Escher's rendering of flying fish. Credit: Institute For Figuring 


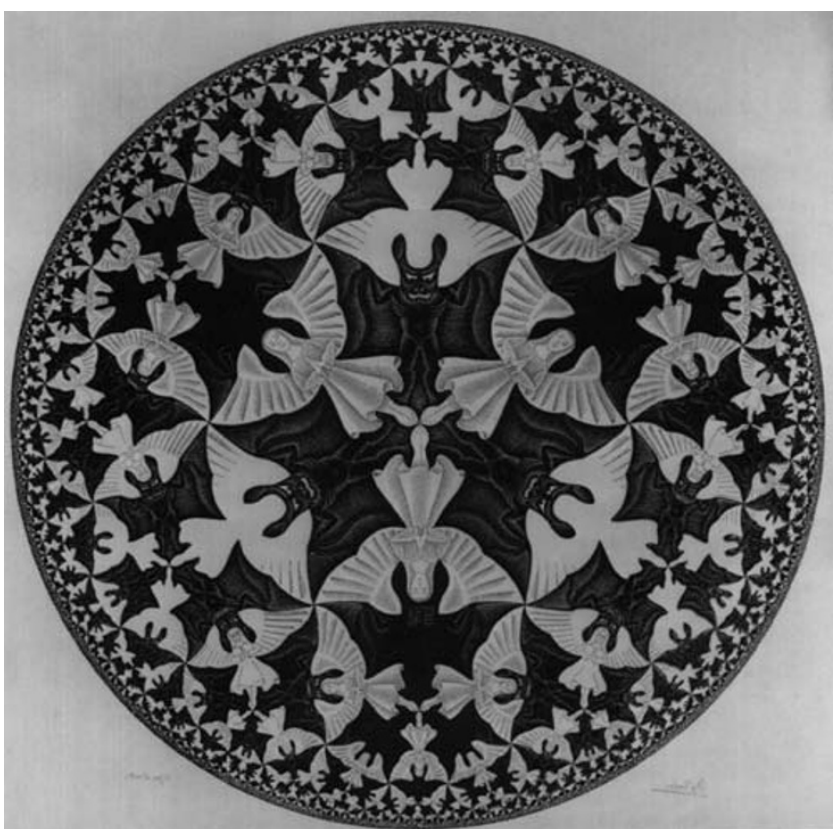

Fig. 6 Circle Limit IV, M.C. Escher's rendering of angels and demons. Credit: Institute For Figuring

For instance, geodesics, or straight lines, can be sewn onto the crochet texture for easy examination. Though the stitched lines in the model appear curved, folding along them demonstrably produces a straight edge. Here, it is obvious that the Euclidean parallel postulate is violated. In the model shown, there are three straight lines that pass through a point external to the bottom line. None of these upper lines ever intersects this original line. Handling this construction, you can physically fold along each line and verify materially that Euclid's axiom does not necessarily hold (Fig. 7).

What insights about evolution can we draw from hyperbolic space theory?

Taimina's crochet techniques allow us to make a great variety of hyperbolic constructions by varying the rate of increase of stitches. If you stick to mathematical perfection you don't get a lot of variation, but if you deviate a little from the perfect geometry, you get this huge variety of forms. And that's really what nature is doing. Just as you don't get perfect spheres or circles in the natural world, neither do you get perfect hyperbolic planes. But the variety of deviations is a great deal richer and more interesting, and this is what we can model with crochet.

Crochet models of hyperbolic space come in a Darwinian variety of shapes and forms. Some resemble kales and kelps, others evoke the animal magnetism of sea slugs or anemones, while others still call to mind the abstractions of the Sydney Opera House. Surfaces may be delicate and lacy or stiff and meaty, fabricated with a needle-fine hook using threads of cotton and silk, or solidly built from thick

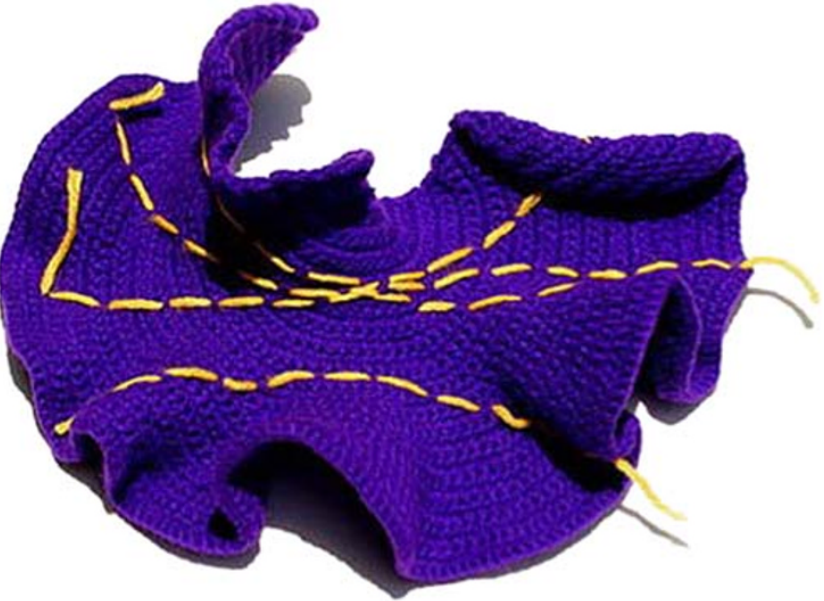

One of the oddest properties of the hyperbolic plane is that there are infinitely many lines passing through a given point parallel to a given line. Here we see three, but there are infinitely many we could draw. On a regular, or euclidean, plane there is only ever one line parallel to a given line that passess through a given point.

Fig. 7 Fold lines indicating parallel lines on a crochet rendering of hyperbolic space. Credit: Institute For Figuring

worsted wools and ropey synthetics. The choice of yarn, the size of hook, the style of stitch and, most importantly, the rate of increase of the stitches all affect the finished model. Though the process is strictly algorithmic, the possible variations are infinite. One really amazing parallel here is that this is really like varying the DNA code of living things - with slight shifts in the DNA, you can get big changes in the morphology of the organism, and the same thing happens when you vary the code or algorithm underlying these crochet forms (Figs. 8, 9, and 10).

There is a very good reason why marine organisms take on a vast variety of hyperbolic forms: this geometry realizes surfaces with maximal area in a limited volume, thus

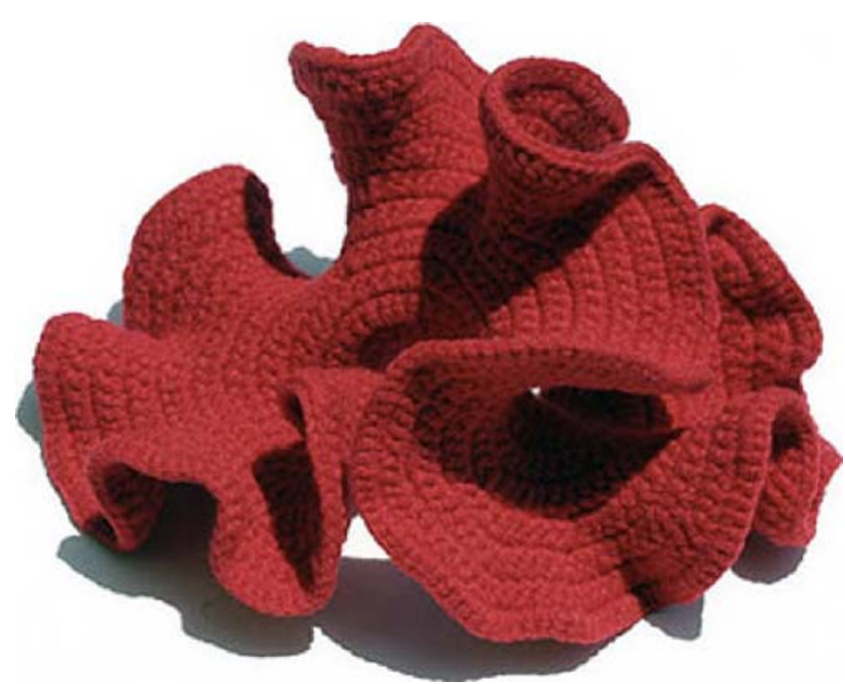

Fig. 8 Crocheted renderings of hyperbolic space models formed with differing stitch increases (compare Figs. 9, 10). Credit: Institute For Figuring 


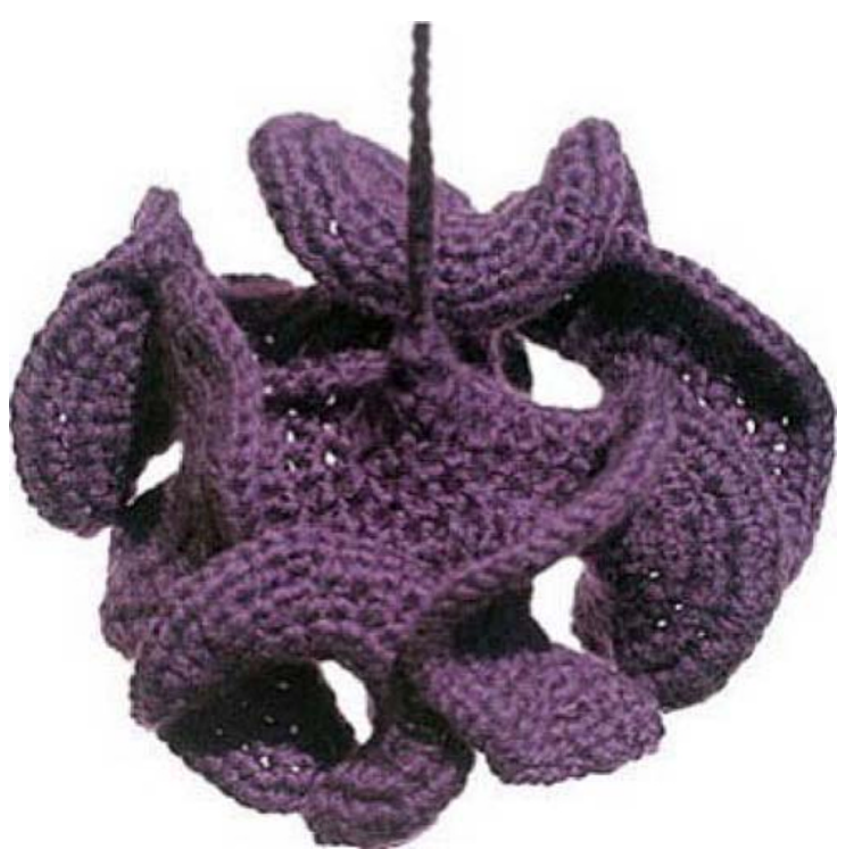

Fig. 9 Crocheted renderings of hyperbolic space models formed with differing stitch increases (compare Figs. 8, 10). Credit: Institute For Figuring

providing enhanced opportunity for filter feeding by sessile creatures.

My organization, Institute For Figuring, has been extending the techniques developed by Dr Taimina-she really sticks with the perfectly geometric forms, whereas we've been branching out to explore the more organic forms that arise when you relax from that perfection. Over the past 3 years, we've had lots of workshops and taught other people to do this, and we find that everyone who takes this up seriously seems to develop their own variations - often, things that we had not thought of. So it has become like a giant world-wide experiment in practical evolution. In a very real sense, our crochet reef

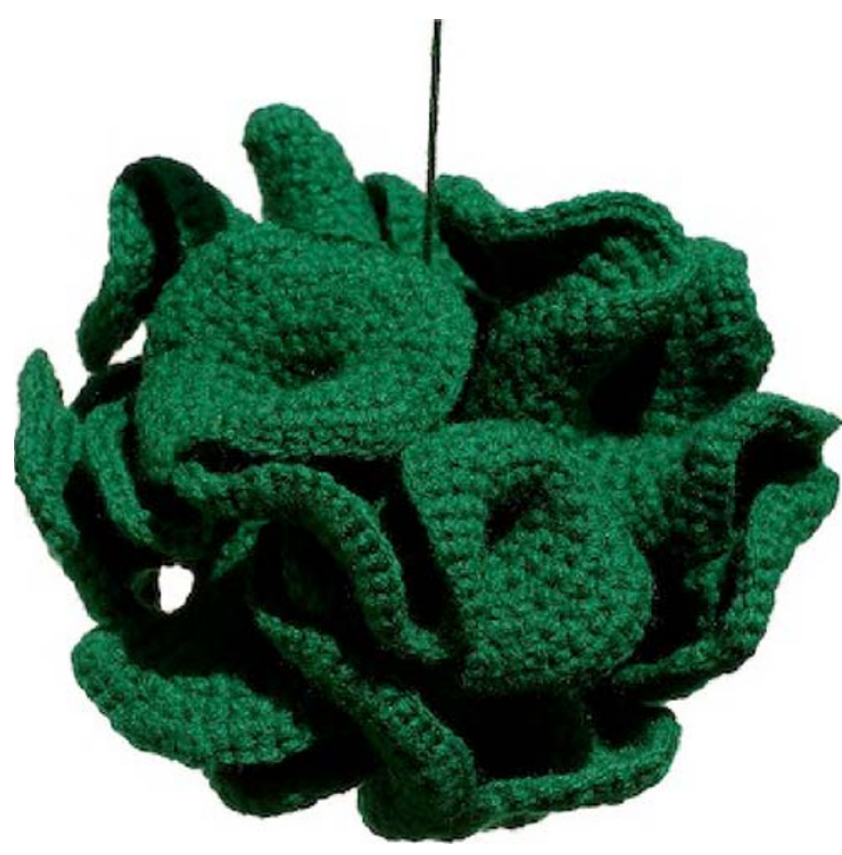

Fig. 10 Crocheted renderings of hyperbolic space models formed with differing stitch increases (compare Figs. 8, 9). Credit: Institute For Figuring

organisms are evolving. Now we've got a very wide taxonomy of hyperbolic crochet "species" and the range of possible forms seems to be endless.

In closing, does a knowledge of hyperbolic space inform your spiritual views?

Escher was inspired by his explorations of hyperbolic space to visit the Alhambra Palace, in Spain, the apotheosis of the Arab world's incredible tradition of work in the medium of tile. Now the Moors believed that repeated patterns connote the divine, so, if you follow that line of thought, you might conclude that Heaven would be a hyperbolic space.

More information about the crochet reef project can be seen on the IFF website at www.theiff.org. 